\title{
A case of lung adenocarcinoma with a concurrent EGFR mutation and ALK rearrangement: A case report and literature review
}

\author{
CHUN-WEI XU ${ }^{1 *}$, XUE-YING CAI ${ }^{2 *}, Y_{U A N}$ SHAO $^{3}$, YANG LI $^{4}$, MING-WEI SHI ${ }^{4}$, \\ LI-YING ZHANG $^{1}$, LIN WANG ${ }^{1}$, YU-PING ZHANG ${ }^{1}$, LU-PING WANG ${ }^{1}$ and YU-WANG TIAN ${ }^{1}$ \\ ${ }^{1}$ Department of Pathology, The General Military Hospital of Beijing PLA, Beijing 100700; ${ }^{2}$ Department of Internal Medicine, \\ Shaoxing People's Hospital, Shaoxing Hospital of Zhejiang University, Shaoxing, Zhejiang 312000; \\ ${ }^{3}$ Department of Surgical Medicine, The First Affiliated Hospital of Xi'an Jiaotong University, Xi'an, Shaanxi 710061; \\ ${ }^{4}$ Department of Oncology, The General Military Hospital of Beijing PLA, Beijing 100700, P.R. China
}

Received August 3, 2014; Accepted May 6, 2015

DOI: $10.3892 / \mathrm{mmr} .2015 .4001$

\begin{abstract}
The echinoderm microtubule associated protein like 4-anaplastic lymphoma kinase (EML4-ALK) fusion is almost mutually exclusive to epidermal growth factor receptor (EGFR) or K-RAS mutation in non-small cell lung cancer (NSCLC), and it is extremely rare for patients to exhibit both mutations. The present study reported the case of a 71-year-old female diagnosed with adenocarcinoma, exhibiting mutations in EGFR and EML4-ALK. The present study treated this patient with EGFR-TK inhibitors, as the first line therapy, and gefitinib therapy revealed a good response until now. In addition, previously reported cases and associated literature were reviewed. The present study provided a greater understanding of the molecular biology and optimal treatment for patients with NSCLC with $>1$ driver mutation.
\end{abstract}

\section{Introduction}

Lung cancer is one of the most common types of malignant tumor worldwide, of which non-small cell lung cancer (NSCLC) accounts for $>85 \%$. The trend of lung cancer treatment has been towards personalized therapy and molecular targeted therapy is currently one of the most popular and promising fields of advanced NSCLC treatment. Driver genes, including epidermal growth factor receptor (EGFR) and echinoderm microtubule associated protein like 4-anaplastic

Correspondence to: Professor Li-Ying Zhang, Department of Pathology, The General Military Hospital of Beijing PLA, 5 Dongsishitiao Street, Beijing 100700, P.R. China

E-mail: lyzhang1@126.com

*Contributed equally

Key words: non-small cell lung carcinoma, epidermal growth factor receptor gene mutation, echinoderm microtubule associated protein like 4-anaplastic lymphoma kinase fusion gene, adenocarcinoma, gefitinib lymphoma kinase (EML4-ALK) are common target genes, and the success of the clinical application of inhibitors against these two molecular targets have been demonstrated in East Asia $(1,2)$. Previous studies suggested that the EML4-ALK fusion is almost mutually exclusive to EGFR or K-RAS mutation in NSCLC (3-5). However, at least 11 patients exhibiting both an EGFR mutation and the EML4-ALK fusion have been reported worldwide (6-13). The present study reports a twelfth case and also the first case of a patient of northern Han Chinese ethnicity who exhibits the two concomitant mutations. The most effective treatment for these two gene-positive patients remains to be elucidated with discordant results reported previously in the literature. All protocols in the present study were approved by the Human Clinical and Research Ethics Committees of the First Affiliated Hospital of Xi'an Jiaotong University (Xi'an, China) and the General Military Hospital of Beijing PLA (Beijing, China). The patient provided written informed consent.

\section{Case report}

A 71 year-old female who has never smoked and originates from Tongchuan City (Shan'xi, China), was first admitted to The First Affiliated Hospital of Xi'an Jiaotong University (Xi'an, China) as a result of left leg pain and swelling, without fever, in March 2013. Imaging examination, including computed tomography (CT) and magnetic resonance imaging (MRI), revealed a $40 \mathrm{~mm}$ tumor in her left lower lung (Fig. 1A) and left tibia destruction with a soft tissue mass (Fig. 2A). No significant previous medical history and laboratory findings were reported. The surgery on the left tibia was performed with the pathological diagnosis of poorly differentiated metastatic adenocarcinoma of the tibia from the left lower lung, clinical stage IV (cT3N3M1b; Fig. 3A and $\mathrm{B}$ ). Following diagnosis, the patient was referred to The General Military Hospital of Beijing PLA (Beijing, China) in April 2013. A further lung biopsy was not performed considering the patient's clinical status. Gene detection for mutations in EGFR (Fig. 4A) and EML4-ALK (Fig. 4B) was performed on a formalin-fixed, paraffin-embedded tibia tumor specimen, by reverse transcription-quantitative 


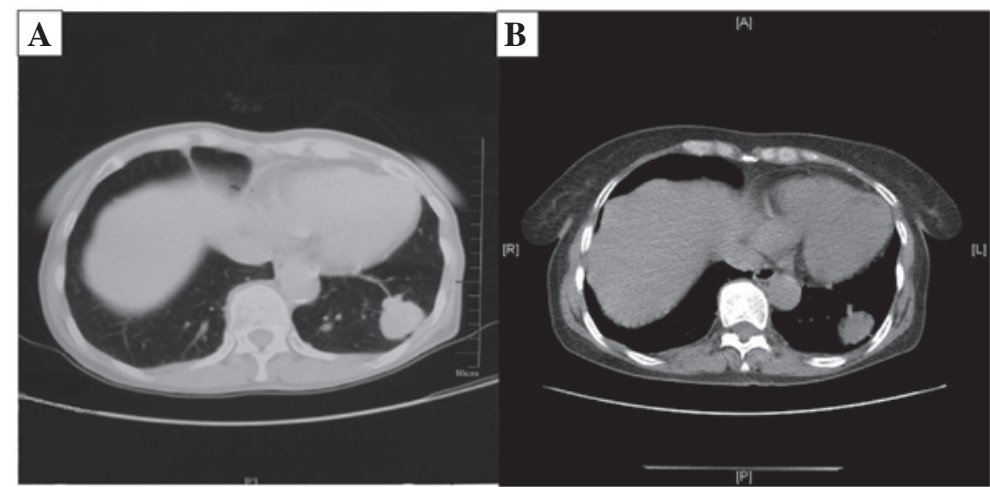

Figure 1. Initial CT scan of the thorax and following treatment with gefitinib. CT scans were captured in the First Affiliated Hospital of Xi'an Jiaotong University (A) prior to treatment and in the General Military Hospital of Beijing PLA (B) 186 days after treatment with gefitinib. CT, computed tomography.

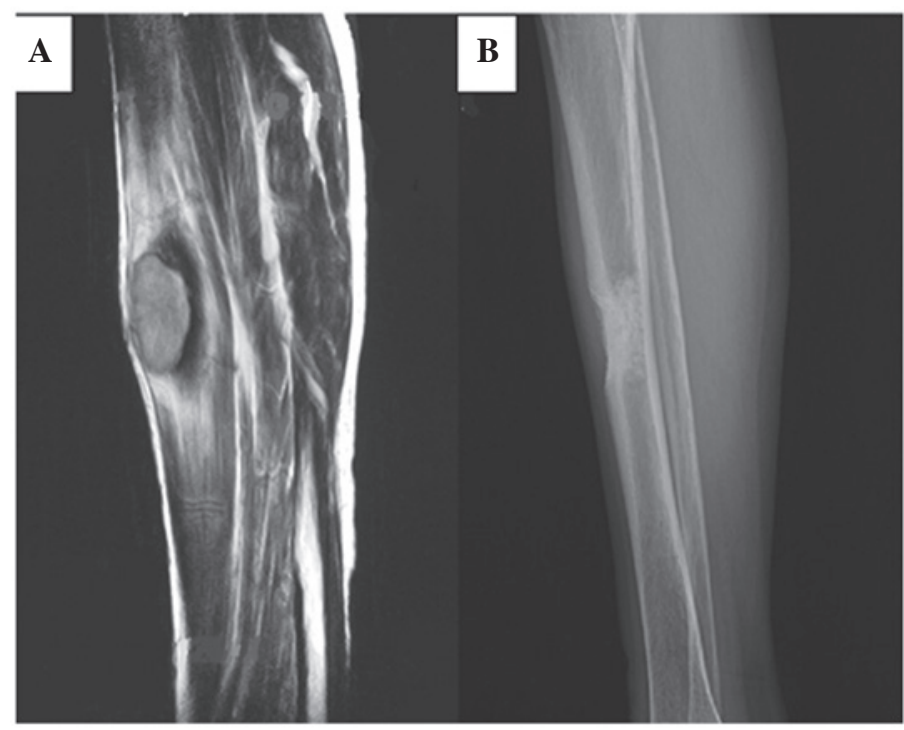

Figure 2. Initial MRI scan of the leg and following treatment with gefitinib. MRI scans were captured in the First Affiliated Hospital of Xi'an Jiaotong University (A) prior to and an X-ray plain film was captured at the General Military Hospital of Beijing PLA (B) following after treatment with gefitinib. MRI, magnetic resonance imaging.

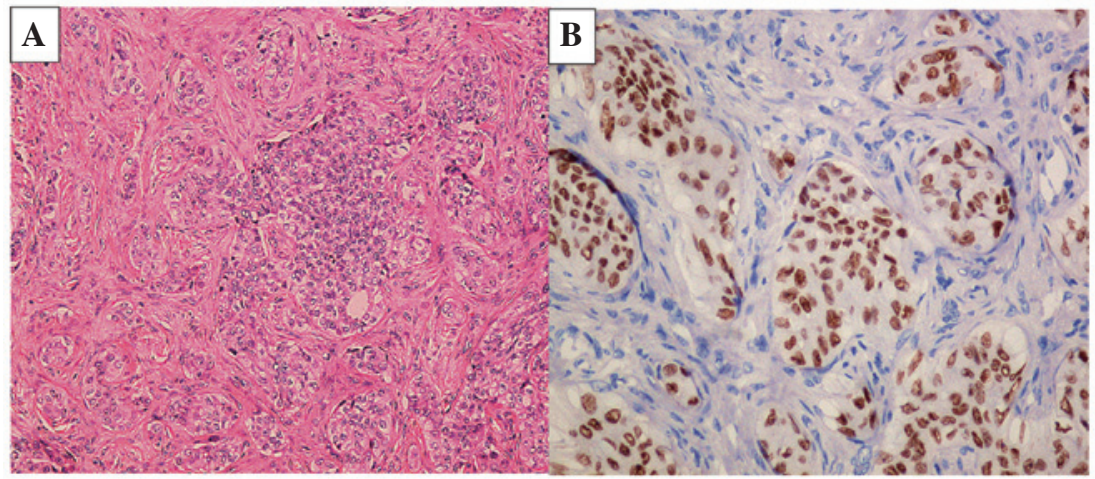

Figure 3. Histology of the bone metastasis. (A) The hematoxylin-eosin staining revealed that tumor cells were transformed lung adenocarcinoma cells (magnification, x200). (B) Immunohistochenmical examination revealed that the tumor cells were positive for staining with the monoclonal anti-TTF-1 antibody (magnification, x200).

polymerase chain reaction. The genomic DNA was extracted using QIAamp DNA FFPE Tissue kit (Qiagen, Inc., Hilden, Germany) and the total RNA was extracted using an RNeasy Mini kit (Qiagen, Inc.), and was reverse-transcribed into
cDNA using RevertAid ${ }^{\mathrm{TM}}$ First Strand cDNA Synthesis kit (Fermentas, Thermo Scientific, Wilmington, DE, USA). The human EGFR mutation qualitative detection kit was the EGFR ADx-ARMS kit and the human EML4-ALK gene expression 


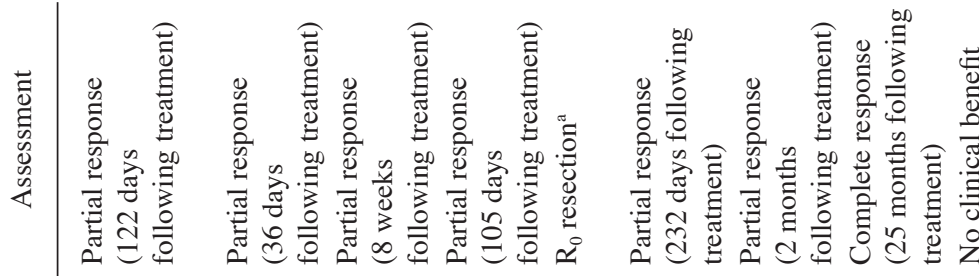

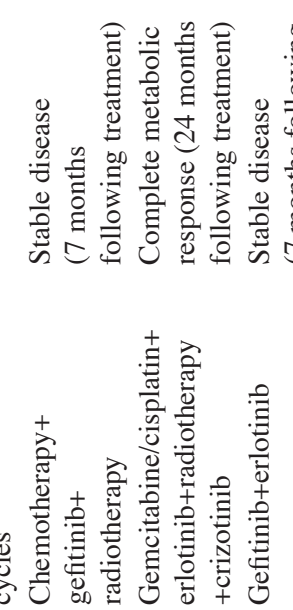

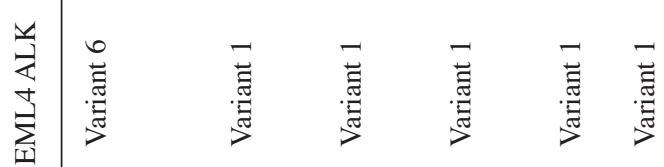

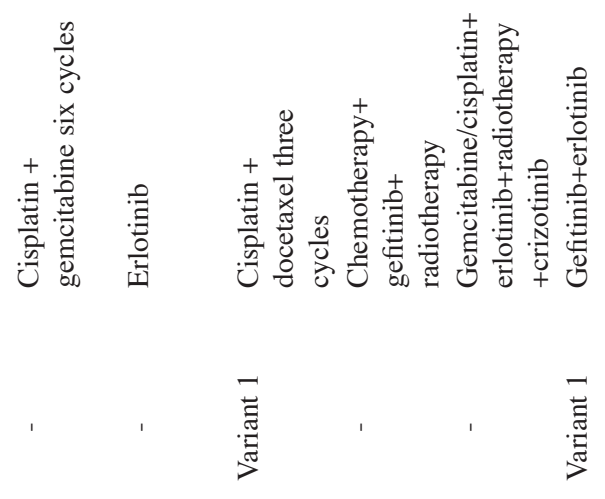

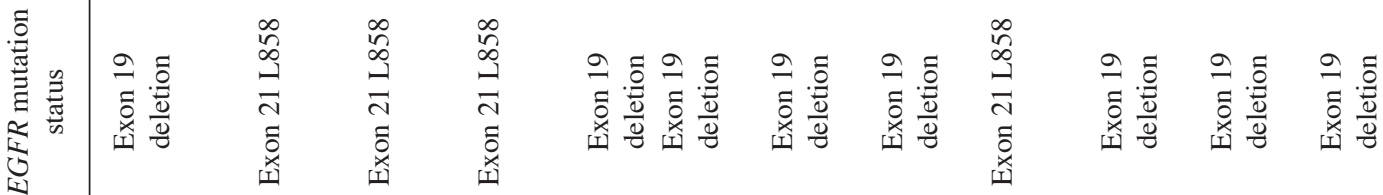

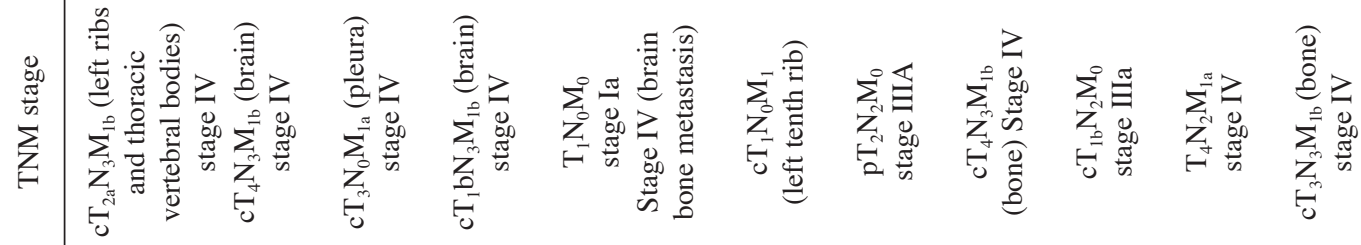

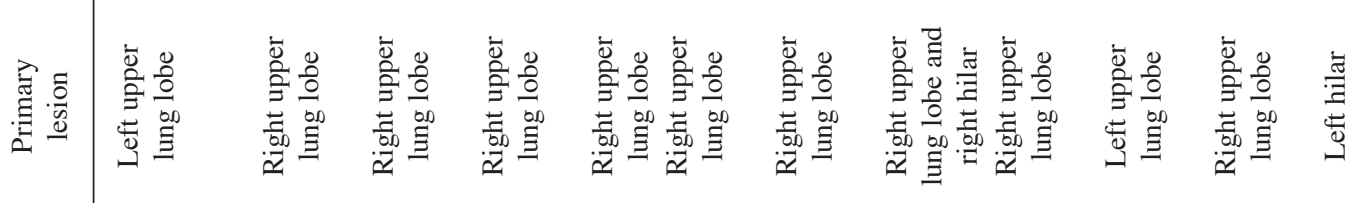

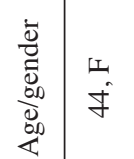
$\begin{array}{ll}I_{1} & \Sigma \\ i & 0\end{array}$ $\Sigma$ in

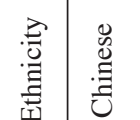

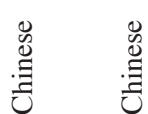

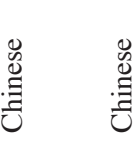
造离

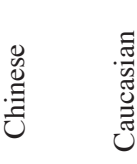
हี 

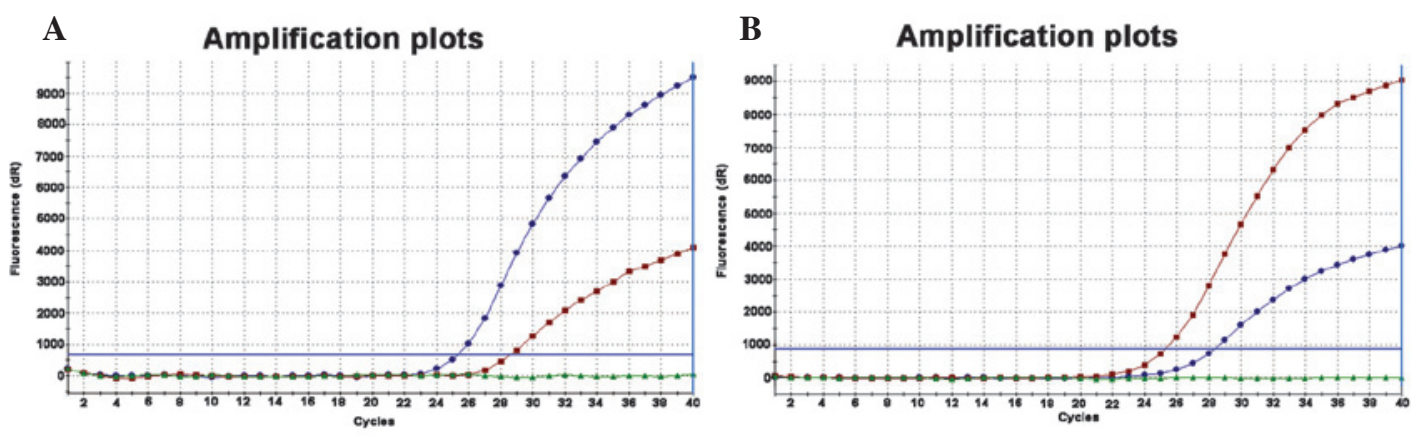

Figure 4. (A) EGFR19 exon amplification curve and (B) EML4-ALK amplification curve results. The blue, red and green represent the sample, positive control and negative control, respectively. EML4-ALK, echinoderm microtubule associated protein like 4-anaplastic lymphoma kinase; EGFR, epidermal growth factor receptor.

A 50

60

70

80

90

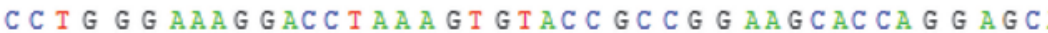

B
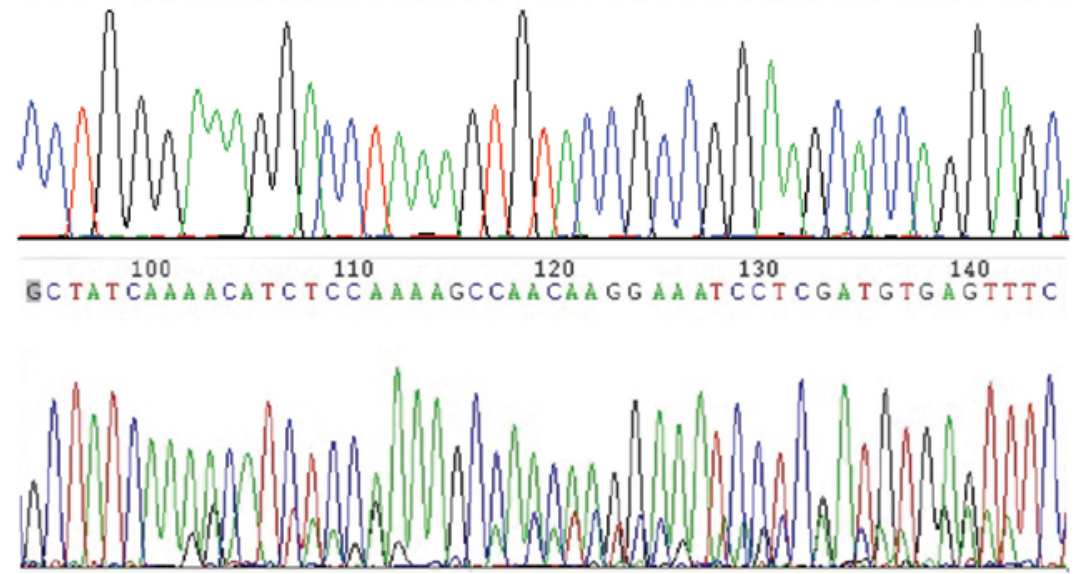

Figure 5. (A) Variant 1 of EML4-ALK translocation in bone metastasis. (B) Deletion in EGFR exon 19 (heterozygous 2235_2249del15; E746_A750del) in bone metastasis. EML4-ALK, echinoderm microtubule associated protein like 4-anaplastic lymphoma kinase; EGFR, epidermal growth factor receptor.

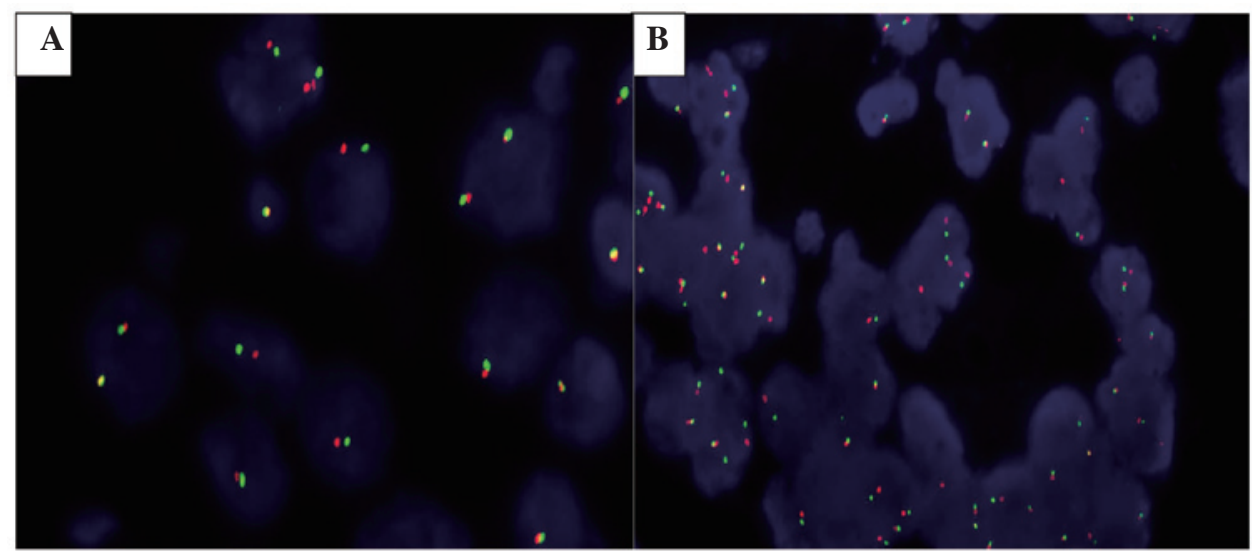

Figure 6. Fluorescent in situ hybridization reveals a split of red and green probes, which flank the ALK translocation site in an EML4-ALK-positive tumor. (A) Large nucleus sections; (B) small nucleus sections. Staining with 4',6-diamidino-2-phenylindole; magnification, x1,000. EML4-ALK, echinoderm microtubule associated protein like 4-anaplastic lymphoma kinase.

assay kit was the EML4-ALK ADx-ARMS kit (Amoy ADx Ltd., Xiamen, China). The amplification products were sent to Beijing Jin Weizhi Biological Technology Co., Ltd. (Beijing, China) for sequencing and a variant 1 of the EML4-ALK translocation (Fig. 5A) and an EGFR exon 19 deletion 2235-2249 (delE746-A750) were detected (Fig. 5B). An ALK rearrangement was further confirmed by fluorescence in situ hybridization using the commercially available breakapart probe (Guangzhou LBP Medicine Science \& Technology Co., Ltd., Guangzhou, China; Fig. 6). The patient was administered $250 \mathrm{mg}$ gefitinib orally every day as the first line chemotherapy and linear accelerator radiotherapy at the left 
tibia by PTV $2 \mathrm{~Gy} / 30 \mathrm{~Gy} / 15 \mathrm{~F} / 23$ days. Following 5 months of EGFR-tyrosine kinase inhibitor (TKI) therapy and 15 radiotherapy sessions, imaging examination demonstrated that the left middle tibia cortex was absent, and the bone mineral density was increased (Fig. 2B). Gefitinib therapy revealed an acceptable response with a repeated chest CT (Fig. 1B), which demonstrated no obviously enlarged lesions or novel lesions following 8 months of therapy.

\section{Discussion}

The EGFR gene, located on the 12-14 region of the short arm of chromosome 7, consists of 28 exons and the majority of mutations were located within exons 19-21 of the tyrosine kinase (TK) domain (14-16). Deletions in exon 19, with the highest mutation rate among the total EGFR mutation, are associated with increased gene expression and TK inhibitor sensitivity $(14,17)$. The EML4-ALK fusion gene was first identified by Soda et al in 2007 (3) in a Japanese patient with NSCLC, which is formed by a small inversion within chromosome $2 p$, and at least 11 different variants have been previously identified, with variants 1 and 3 being the most common (3-6,16,18-23).

EGFR-TK inhibitors, gefitinib and erlotinib, have been widely used for the treatment of patients with advanced NSCLC exhibiting the EGFR mutation. Markedly improved benefits were observed from EGFR-TK inhibitors in a previous study, which compared the efficacy of conventional cytotoxic chemotherapy as first-line treatment (24). In the 2012 edition of the NCCN clinical practice guidelines of NSCLC, EGFR mutation detection was suggested in the initial treatment of patients with advanced NSCLC (25). Patients who harbor the EML4-ALK mutation fail to benefit from EGFR-TKIs $(5,21)$. Crizotinib, an orally bioavailable ALK inhibitor, is recommended for treating these patients (26) and is currently under phase III clinical trials worldwide. Whether EML4-ALK NSCLC can behave in an analogous manner to the EGFR mutant NSCLC and whether crizotinib can become a milestone in the treatment of NSCLC remains to be elucidated.

Patients who exhibit both mutations are extremely rare and previous studies have suggested that an EGFR mutation and EML4-ALK gene fusion are mutually exclusive molecular events $(3-6,18,20-22,27)$. The literature for both the EGFR and EML4-ALK mutations in NSCLC was assessed and revealed only 12 cases, including the present case (Table I). This is also the first case, to the best of our knowledge, in the northern Han Chinese population identified with a concurrent EGFR exon 19 deletion 2235-2249 (delE746-A750) and EML4-ALK variant 1. As shown in Table I, the clinical characteristics of the 12 cases of patients with advanced stage NSCLC with the concomitant mutations presented in our study were as follows: Median age, 57; 7/12 female; 9/12 Asian (8/12 Chinese, 1/12 Japanese); 9/12 light-smoker or never-smoked; pathological type, 11/12 adenocarcinoma and 1/12 adenosquamous carcinoma; and 8/12 EGFR exon 19 or 4/12 exon 21 mutation, coexisted with the EML4-ALK variant 1 (7/8) or variant $6(1 / 8)$. A favorable response was observed according to image analysis following treatment with gefitinib for 8 months in the present case study. However, the results reported in the literature are inconsistent and the appropriate treatment for this subset of patients with NSCLC remains to be elucidated.

Finally, the present study had certain limitations. Firstly, the primary lung tumor specimen is no longer available, therefore, our results cannot be further verified in the primary tumor, with the possible mechanism of drug resistance, including primary drug resistance and acquired resistance remaining unknown. Secondly, the response of the ALK inhibitor in this patient is unknown since no ALK-targeted agents were used.

In conclusion, the present study reported a rare case of lung cancer, harboring both the EGFR mutation and the EML4-ALK fusion gene. Treatment with gefitinib has demonstrated a good response thus far. Future research and experience are required to understand the biological features and the optimal targeted treatment modes for this subtype of patients with lung cancer.

\section{References}

1. Zhou Q, Zhou CC, Chen GY, et al: A multicenter phase II study of sorafenib monotherapy in clinically selected patients with advanced lung adenocarcinoma after failure of EGFR-TKI therapy (Chinese Thoracic Oncology Group, CTONG 0805). Lung Cancer 83: 369-373, 2014.

2. Wang Z, Zhang X, Bai H, et al: EML4-ALK rearrangement and its clinical significance in Chinese patients with advanced non-small cell lung cancer. Oncology-83: 248-256, 2012.

3. Soda M, Choi YL, Enomoto M, et al: Identification of the transforming EML4-ALK fusion gene in non-small-cell lung cancer. Nature 448: 561-566, 2007.

4. Inamura $\mathrm{K}$, Takeuchi $\mathrm{K}$, Togashi $\mathrm{Y}$, et al: EML4-ALK fusion is linked to histological characteristics in a subset of lung cancers. J Thorac Oncol 3: 13-17, 2008.

5. Koivunen JP, Mermel C, Zejnullahu K, et al: EML4-ALK fusion gene and efficacy of an ALK kinase inhibitor in lung cancer. Clin Cancer Res 14: 4275-4283, 2008.

6. Yang J, Zhang X, Su J, et al: Concomitant EGFR mutation and EML4-ALK gene fusion in non-small cell lung cancer. J Clin Oncol 29 (Suppl): [abstr 10517], 2011.

7. Zhang X, Zhang S, Yang X, et al: Fusion of EML4 and ALK is associated with development of lung adenocarcinomas lacking EGFR and KRAS mutations and is correlated with ALK expression. Mol Cancer 9: 188, 2010.

8. Kuo YW, Wu SG, Ho CC, et al: Good response to gefitinib in lung adenocarcinoma harboring coexisting EML4-ALK fusion gene and EGFR mutation. J Thorac Oncol 5: 2039-2040, 2010.

9. Tiseo M, Gelsomino F, Boggiani D, et al: EGFR and EML4-ALK gene mutations in NSCLC: A case report of erlotinib-resistant patient with both concomitant mutations. Lung Cancer 71 241-243, 2011.

10. Popat S, Vieira de Araújo A, Min T, et al: Lung adenocarcinoma with concurrent exon 19 EGFR mutation and ALK rearrangement responding to erlotinib. J Thorac Oncol 6: 1962-1963, 2011.

11. Tanaka H, Hayashi A, Morimoto T, et al: A case of lung adenocarcinoma harboring EGFR mutation and EML4-ALK fusion gene. BMC Cancer 12: 558, 2012.

12. Santelmo C, Ravaioli A, Barzotti E, et al: Coexistence of EGFR mutation and ALK translocation in NSCLC: Literature review and case report of response to gefitinib. Lung Cancer 81: 294-296, 2013.

13. Chen $\mathrm{X}$, Zhang $\mathrm{J}, \mathrm{Hu} \mathrm{Q}$, et al: A case of lung adenocarcinoma harboring exon 19 EGFR deletion and EML4-ALK fusion gene. Lung Cancer 81: 308-310, 2013.

14. Shigematsu H, Lin L, Takahashi T, et al: Clinical and biological features associated with epidermal growth factor receptor gene mutations in lung cancers. J Natl Cancer Inst 97: 339-346, 2005.

15. Kosaka T, Yatabe Y, Endoh H, et al: Mutations of the epidermal growth factor receptor gene in lung cancer: Biological and clinical implications. Cancer Res 64: 8919-8923, 2004.

16. Li Y, Li Y, Yang T, et al: Clinical significance of EML4-ALK fusion gene and association with EGFR and KRAS gene mutations in 208 Chinese patients with non-small cell lung cancer. PLoS One 8: e52093, 2013. 
17. Gazdar AF, Shigematsu H, Herz J, et al: Mutations and addiction to EGFR: The Achilles 'heal' of lung cancers? Trends Mol Med 10: 481-486, 2004

18. Inamura $\mathrm{K}$, Takeuchi $\mathrm{K}$, Togashi $\mathrm{Y}$, et al: EML4-ALK lung cancers are characterized by rare other mutations, a TTF-1 cell lineage, an acinar histology and young onset. Mod Pathol 22: 508-515, 2009.

19. Martelli MP, Sozzi G, Hernandez L, et al: EML4-ALK rearrangement in non-small cell lung cancer and non-tumor lung tissues. Am J Pathol 174: 661-670, 2009.

20. Shaw AT, Yeap BY, Mino-Kenudson M, et al: Clinical features and outcome of patients with non-small-cell lung cancer who harbor EML4-ALK. J Clin Oncol 27: 4247-4253, 2009.

21. Wong DW, Leung EL, So KK, et al: The EML4-ALK fusion gene is involved in various histologic types of lung cancers from nonsmokers with wild-type EGFR and KRAS. Cancer 115: $1723-1733,2009$.

22. Takahashi T, Sonobe M, Kobayashi M, et al: Clinicopathologic features of non-small-cell lung cancer with EML4-ALK fusion gene. Ann Surg Oncol 17: 889-897, 2010.
23. Takeda M, Okamoto I, Sakai K, et al: Clinical outcome for EML4-ALK-positive patients with advanced non-small-cell lung cancer treated with first-line platinum-based chemotherapy. Ann Oncol 23: 2931-2936, 2012.

24. Mok TS, Wu YL, Thongprasert S, Yang CH, Chu DT, Saijo N, Sunpaweravong P, Han B, et al: Gefitinib or carboplatin-paclitaxel in pulmonary adenocarcinoma. N Engl J Med 361: 947-957, 2009.

25. Zornosa C, Vandergrift JL, Kalemkerian GP, et al: First-line systemic therapy practice patterns and concordance with NCCN guidelines for patients diagnosed with metastatic NSCLC treated at NCCN institutions. J Natl Compr Canc Netw 10: 847-856, 2012.

26. Camidge DR, Bang YJ, Kwak EL, et al: Activity and safety of crizotinib in patients with ALK-positive non-small-cell lung cancer:updated results from a phase I study. Lancet Oncol 13: 1011-1019, 2012.

27. Sasaki T, Rodig SJ, Chirieac LR and Jänne PA: The biology and treatment of EML4-ALK non-small cell lung cancer. Eur J Cancer 46: 1773-1780, 2010. 